\title{
Hommage
}

\section{Jean-Pierre Deffontaines 1933-2006}

ean-Pierre Deffontaines, géoagronome Inra, membre de l'Académie d'agriculture, nous a quittés le 25 octobre 2006 . Il est né à Cusance, dans le Doubs, le 17 juillet 1933. Il a fait ses études secondaires à Barcelone (Espagne), alors que son père était directeur de l'Institut français de Barcelone, puis des études préparatoires au Lycée Saint-Louis à Paris. Il a ensuite intégré l'Institut national agronomique en 1957 où il a eu pour maittres René Dumont et Stéphane Hénin : il doit à l'un le souci du développement local dans ses rapports au mondial, à l'autre l'attention aux techniques et pratiques mises en œuvre par les agriculteurs eux-mêmes. À la sortie de l'Agro, il fait un séjour en Argentine où il va développer un regard anthropologique sur l'activité agricole. Après 24 mois de guerre en Algérie, il est recruté comme assistant à l'Agro et travaille de 1961 à 1965 sous la direction de Stéphane Hénin. En 1966, il soutient une thèse de géographie. Cette même année, il devient chargé de recherche au Service d'expérimentation et d'information (SEI) de l'Institut national de la recherche agronomique (Inra) à Versailles, puis directeur de recherche en 1984. Il dirige l'unité SAD ${ }^{1}$ de Versailles jusqu'en 1998. En 1996, il devient membre de l'Académie d'agriculture.

Géoagronome, c'est le terme qu'il avait adopté pour se définir : un chercheur agronome intégrant la préoccupation territoriale du géographe "dans une perspective de développement local ", un géographe mettant l'accent sur l'importance du fait "biotechnique ", comme constituant intime des territoires ruraux. Non pas un écartèlement entre le géographe et l'agronome, mais une fusion originale obtenue par interactions successives, renouvelant l'approche du géographe par la connaissance des terrains et celle de l'agronome par la dimension territoriale.

Jean-Pierre Deffontaines va participer à la formulation du principe que "les agriculteurs ont de bonnes raisons de faire ce qu'ils font ". Une inflexion majeure en matière de conception du développement agricole. Elle justifie, ce qui est alors improprement appelé "la résistance au changement " face au bulldozer de la modernisation de l'agriculture. Ce qu'il met en cause, ce n'est pas le principe même de la modernisation, ce sont les modalités et les mots d'ordre qui ne prennent pas en compte la diversité des situations personnelles, la réalité du "système famille-exploitation ". Avec le recul des ans, un tel principe donne de la légitimité à la diversité des choix qui sont ceux du monde agricole aujourd'hui. De plus, il a l'intuition féconde et prémonitoire que les agricultures des régions dites « en retard de développement " ou à "faibles potentialités " méritent une attention particulière parce qu'elles sont riches de questionnements pour l'ensemble de l'agriculture, y compris celle des régions " riches " - Vosges, Corse, Causses - c'était il y a trente ans.

Quelles sont les conséquences de cet "individualisme "sur le développement des territoires ruraux? Cette question va prendre une place considérable dans ses travaux. Les équipes multidisciplinaires qu'il constitue et anime accordent autant d'importance aux typologies des exploitations agricoles - la diversité des exploitations et des situations familiales - qu'aux analyses de paysage. Le paysage est, pour Jean-Pierre Deffontaines, non pas une finalité, mais l'expression visuelle et le révélateur des évolutions sociales, économiques et environnementales de l'agriculture et de ses travaux aux champs. "Pays, paysans, paysages dans les Vosges du Sud" constitue l'ouvrage de référence de cette démarche. Un retour à la définition première de l'agronome qui combine la fonction d'" inspecteur des champs et des campagnes " et celle de " théoricien de l'agriculture ". Une projection nouvelle faisant appel à l'analyse systémique et contribuant aux sciences de la gestion.

Il faut bien dire qu'au sein de l'Inra, cette posture de recherche rencontre alors l'incrédulité de ceux qui croient aux seules vertus de la formation des agriculteurs aux technologies performantes issues de la recherche agronomique, qui ne comprennent pas que l'on puisse attacher de l'intérêt aux pratiques des agriculteurs, qui considèrent que l'agriculture de plaine est la seule qui vaille attention. Elle va trouver sa reconnaissance à la fin des années 1970 avec la création, controversée, mais décidée par le directeur général de l'époque, Jacques Poly, d'un nouveau département de recherche, le SAD. Jean-Pierre Deffontaines y apporte son expérience de terrain et sa capacité d'élaboration conceptuelle, avec d'autres équipes aux démarches tout aussi atypiques. Non sans révolte de sa part face aux contraintes administratives, non sans vivacité face aux incompréhensions, non sans énergie pour emporter l'adhésion. Parmi d'autres exemples, le cas de Vittel, avec l'adoption de pratiques agricoles assurant la protection d'un périmètre de captage d'eau de source, démontre, si cela était nécessaire, combien est fructueuse cette nouvelle démarche de la recherche agronomique.

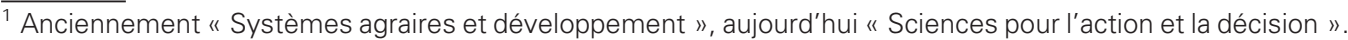


Jean Pierre Deffontaines a toujours manifesté un grand intérêt pour les situations hors hexagone, en particulier en Amérique latine. Entre 1990 et 1994, il a participé à un programme de coopération entre l'Inra et l'Instituto Nacional de Tecnología Agropecuaria (Inta) où il a mis à l'épreuve l'analyse du paysage et la méthode des UAP et UAG (unité agrophysionomique et unité agrogéographique) pour définir des " territoires de développement ". Un éleveur, qui l'avait reçu lors de son premier séjour, se souvenait encore de Jean-Pierre comme d'un agronome s'intéressant aussi bien aux plantes et aux animaux qu'aux paysages et aux hommes.

Cette expérience, cette vision conceptuelle du paysage, il en a fait profiter les Cabiers Agricultures. Membre du comité scientifique, il avait en juillet dernier accepté de poursuivre son action à la condition que sa santé le lui permette.

En avril dernier, face au vallon de Clairvaux-d'Aveyron, "Deff " donnait aux membres du Groupe de Camboulazet et aux étudiants du lycée agricole de La Roque, ce qui allait être sa dernière "leçon de paysage ", avec la minutie qui était la sienne, son souci du repérage du détail significatif au sein du tout, son écoute aux enjeux nouveaux auxquels les acteurs du paysage étaient confrontés, sa réceptivité chaleureuse aux questionnements qui surgissaient ${ }^{2}$. C'était au pays de Farrebique!

Jean-Claude Flamant 2 novembre 2006

\footnotetext{
${ }^{2}$ Un ouvrage accompagné d'une vaste bibliographie rassemble les textes les plus significatifs de Jean-Pierre Deffontaines: Les sentiers d'un
} géoagronome, éditions Arguments, 1998. 